\title{
Robotic-assisted laparoscopic radical cystectomy: Surgical and oncological outcomes
}

\author{
Adrian Treiyer, Matthias Saar, Zentia Bütow, Jörn Kamradt, Stefan Siemer, Michael Stöckle \\ Division of Robotic Urology, Saarland University, Homburg/Saar, Germany
}

\section{ABSTRACT}

Purpose: Our first 91 consecutive cases undergoing a robotic assisted cystectomy were analyzed regarding perioperative outcomes, pathological stages and surgical complications.

Materials and Methods: Between 2007 and 2010 a total of 91 patients (76 male and 15 female), 86 with clinically localized bladder cancer and 5 with non-urothelial tumors underwent a radical robotic assisted cystectomy. We analyzed the perioperative factors, length of hospital stay, pathological outcomes and complication rates.

Results: Mean age was 65.6 years (range 28 to 82). Among the 91 patients, 68 were submitted to an ileal conduit and 23 to a neobladder procedure for urinary diversion. Mean operating time was 412 min (range: 243-618 min.) and mean blood loss was $294 \mathrm{~mL}$ (range: 50-2000 mL). In 29\% of the cases with urothelial carcinoma the T-stage was pT1 or less, 38\% were pT2; $26 \%$ and $7 \%$ were classified as pT3 and pT4, respectively. 14\% of cases had lymph node positive disease. Mean number of lymph nodes removed was 15 (range 4 to 33). Positive surgical margins occurred in 2 cases $(2.1 \%)$. Mean days to flatus were 2.13 , bowel movement 2.88 and inpatient stay 18.8 (range: 10-33). There were 45 postoperative complications with $11 \%$ major (Clavien grade 3 or higher). At a mean follow-up of 15 months 10 patients had disease recurrence and 6 died of the disease.

Conclusions: Our experience demonstrates that robotic assisted radical cystectomies for the treatment of bladder cancers seems to be very promising regarding surgical and oncological outcomes.

\section{ARTICLE INFO}

\section{Key words:}

Robotics; cystectomy;

bladder; carcinoma

Int Braz J Urol. 2012; 38: 324-9

Submitted for publication:

November 03, 2011

Accepted after revision:

April 18, 2012

\section{INTRODUCTION}

Robotic-assisted laparoscopic radical cystectomy has become a surgical option for patients with bladder cancer, providing the benefits of minimal invasive surgery with lower blood loss, early return of bowel function and more rapid patient recovery, while apparently maintaining functional and oncological outcomes (1-6). The surgical and perioperative outcomes of initial reports appear to be comparable to the open approach. However, larger experiences are required to adequately evaluate and validate this procedure as an appropriate surgical and oncological method for patients with bladder cancer. We report our initial experience with robotic assisted laparoscopic radical cystectomy, evaluating the perioperative and pathological outcomes of this procedure. 


\section{MATERIALS AND METHODS}

All patients underwent preoperative laboratory studies and imaging (chest $\mathrm{x}$-ray and abdominal/pelvic cross-sectional imaging). In all urothelial carcinoma cases a transurethral bladder tumor resection was performed preoperatively. No patients received preoperative neoadjuvant chemotherapy. Table-1 lists patient characteristics.

Table 1 - Patient characteristics.

\begin{tabular}{lc}
\hline Mean Age (range) & $65.6(28-82)$ \\
\hline № gender & 76 \\
$\quad$ Male & 15 \\
$\quad$ Female & 26.7 \\
Mean body mass index $\left(\mathrm{kg} / \mathrm{m}^{2}\right)$ & 2.21 \\
Mean American Society of & \\
Anesthesiologists score & \\
No clinical stage & 37 \\
$\quad$ CT1 or less & 46 \\
CT2 & 3 \\
CT3-T4 & 5 \\
Other tumors & \\
\hline
\end{tabular}

All patients underwent robotic assisted laparoscopic radical cystectomy using the da Vinci ${ }^{\circledR}$ surgical system. Three surgeons, versed in robotic assisted laparoscopic radical prostatectomies, but neither experienced in laparoscopy nor trained in robotic assisted laparoscopy for radical cystectomies, performed the operations. The robotic-assisted laparoscopic approach was used for the cystoprostatectomy and bilateral pelvic lymph node dissection portions of the procedure and for pre-placing urethral anastomotic stitches in orthotopic neobladder cases. After completing the extirpative portion of the procedure the robot was undocked and urinary diversion (ileal conduit or orthotopic ileal neobladder) was performed via a 5 to $8 \mathrm{~cm}$ lower midline incision. The decision as to which urinary diversion would be performed was taken beforehand and individualized, depending on patient age and preferences. The nerve sparing procedure was performed with titanium clips and cold scissor dissection in a manner similar to that used for robotic prostatectomy, as previously described by Menon et al. (7). The robotic assisted laparoscopic pelvic lymphadenectomy involved lymph nodes up to the level of the common iliac vessels (8).

After surgery, all patients were taken to the urology intermediate care unit and underwent routine postoperative support, which included nasogastric tube removal immediately after surgery, prokinetic agents, nonnarcotic analgesics and early diet advancement.

In this series, which presents the learning curve at our center, we evaluated operative variables such as total surgical time (cystectomy, bilateral pelvic lymphadenectomy and urinary diversion) and estimated blood loss. We included aspects of hospital recovery such as time to flatus, bowel movement and hospital discharge including the 30-day complication rate. Furthermore, we analyzed pathological outcomes such as pathological stage, margin status, bladder entry and the number of lymph nodes removed.

Complications were measured using the Clavien classification system (9). This system is well known in the general surgery and urological literature, and helps to report complications across different institutions (10-11).

\section{RESULTS}

The mean age of patients undergoing the robotic procedure was 65.6 years (range: 28-82 years) with a male to female ratio of $5: 1$. The mean American Society of Anesthesiologists score (ASA) was 2.21. The majority of cases (46) were clinical stage T2, followed by cT1 or less (37) and finally cT3 or cT4 (3). The mean clinical follow-up was 15 months (range: 3-44).

In terms of perioperative outcome mean estimated blood loss was $294 \mathrm{~mL}$ (range: 50-2000 $\mathrm{mL}$ ) and mean overall operating time was 265 min. (range: 243-618 min). 
Mean time to flatus and bowel movement was 2.13 days (range: 1-6 days) and 2.88 days (range: 1-7 days), respectively. Pertaining to the date of discharge, 27 patients (29.6\%) were discharged between the $9^{\text {th }}$ and $13^{\text {th }}$ postoperative day (POD), 28 (30.8\%) were discharged between the $14^{\text {th }}$ and $18^{\text {th }}$ POD, $17(18.8 \%)$ between the $18^{\text {th }}$ and $22^{\text {nd }}$ POD and 19 (20.8\%) were discharged after the 22nd POD. The mean inpatient stay was 18.8 days (range: 10-33). In total, 60\% of patients were discharged less than 18 days after surgery. This extended time of stay can be explained by the fact that, as with conventional open cystectomy patients, these patients were only discharged after all catheters, including ureteral stents, had been removed. This usually took place on the $10^{\text {th }}$ and $11^{\text {th }}$ POD. Therefore, the earliest possible discharge of patients with an ileal conduit was on the $12^{\text {th }}$ POD. Patients with an orthotopic neobladder remained in hospital until removal of the indwelling catheter, which usually took place on the $21^{\text {st }}$ POD. Most patients were also given the option of staying in hospital for a few extra days if they had a strong preference of being discharged directly into a rehabilitation program without prior return to home. As so few postoperative complications were seen in the first patients, the current trend in our center is to decrease the inpatient hospital stay. Table- 2 shows the above discussed parameters.

Table-3 lists pathological outcomes. An inadvertent bladder entry did not occur at all. Organ confined disease was found in $67 \%$ of patients. In 29\% of the cases with urothelial carcinoma, the T-stage was pT1 or less, 38\% were pT2, and 26\% and 7\% were classified as pT3 and pT4, respectively. Two patients $(2.1 \%)$ had positive surgical margins. In one case, the positive surgical margin was discovered postoperatively in the urethra after an intraoperative frozen section described it to be negative. The other patient had a known pT4 prostate cancer that was previously been operated, without successful removal of the primary tumor.

Lymph node positive disease was diagnosed in $14 \%$ of the cases. A mean of 15 lymph nodes (range 1 to 33) were removed with a standard dissection up to the bifurcation of the common iliac vessels.
In the early postoperative period (less than 30 days after surgery) 45 patients had complications, including postoperative bleeding, rehospitalization for nausea/ vomiting, ileus, febrile urinary tract infections, deep vein thrombosis, cardiopulmonary complications and occlusion of the neobladder. However, only 10 patients (11\%) had major complications (Clavien Grade III or higher) that needed invasive treatment. One of those patients died a few days after surgery due to an uncontrollable epileptic attack, complicated by cardiac failure.

The 30 day-readmission rate for this case series was 10.9\% (10 of 91 patients).

Table 2 - Surgical and pathological outcomes.

\begin{tabular}{lc} 
No diversion type (\%) & \\
\hline \multicolumn{1}{c}{ Conduit } & $68(74.7)$ \\
$\quad$ Neobladder & $23(25.3)$ \\
$\begin{array}{l}\text { Mean time of surgery (min) } \\
\text { (range) }\end{array}$ & 265 \\
Pathological findings (n (\%)) & $(243-618)$ \\
(Urothelial carcinoma $\mathbf{n}=86)$ & \\
$\quad$ pT1 or less & \\
$\quad$ pT2 & \\
$\quad$ pT3 & $25(29)$ \\
$\quad$ pT4 & $33(38)$ \\
№ removed lymph nodes & $22(26)$ \\
(range) & $6(7)$ \\
№ positive lymph nodes (\%) & 14.48 \\
№ positive surgical margins (\%) & $(1-33)$ \\
Mean days to flatus & $13(14)$ \\
(range) & $2(2.1)$ \\
Mean days to bowel movement \\
(range) \\
$\begin{array}{l}\text { Mean days to discharge home } \\
\text { (range) }\end{array}$ & 2.13 \\
\end{tabular}


Table 3 - Postoperative Clavien complications.

\begin{tabular}{lc}
\hline No patient with postoperative complications (\%) & $45(49.4)$ \\
\hline $\begin{array}{l}\text { No patient with major complications } \\
\text { (Clavien } 3 \text { or higher) (\%) }\end{array}$ & $10(10.9)$ \\
Complications after Clavien classification & \\
I & $14(15.3)$ \\
II & $21(23.1)$ \\
III & $6(6.6)$ \\
IV & $3(3.3)$ \\
V & $1(1.1)$ \\
\hline
\end{tabular}

There were 7 patients (7.6\%) with evidence of recurrence of the disease.

During follow-up 5 patients died of advanced urothelial carcinoma and 1 died of other causes, thus making the overall survival rate of $93.4 \%$ and a disease specific survival rate of $94 \%$.

\section{DISCUSSION}

Our first experiences in the robotic approach for laparoscopic radical cystectomies demonstrate that the pathological results and desired oncological outcomes could be achieved. It has been postulated that lymph node count and positive soft tissue margins might serve as measures of surgical quality for cystectomy. Experts have recommended that a lymph node yield greater than 10 and a positive surgical margin rate of less than 10\% (some even say less than 5\%) are relevant indices of quality and adequacy $(1,12-14)$. In our study, a mean number of 15 lymph nodes were removed and two patients $(2.1 \%)$ had positive surgical margins. In a recent comparison of open and robotic cystectomy by $\mathrm{Ng}$ et al., the robotic surgery was associated with decreased blood loss, equal mean operative time, and an equal positive surgical margin rate and lymph node yield (7.2\% and 17.9 nodes, respec- tively) (15). Haber and Gill reported a 5\% positive surgical margin rate, a mean lymph node yield of 14 nodes and 92\% 5-year cancer specific survival in 37 patients undergoing laparoscopic radical cystectomy (16). As such, the current report adds to a growing body of literature suggesting that in appropriately selected patients minimally invasive radical cystectomy offers equivalent operative and pathological outcomes, and an acceptable intermediate term oncological efficacy.

With regards to perioperative outcomes a mean robotic operating time of $265 \mathrm{~min}$ is comparable with the results published in other studies (1-6,17-20).

Similarly, we described a low surgical blood loss that contributes to a faster postsurgical recovery as was demonstrated in other robotic cystectomy reports (1-6,17-20).

In addition, postoperative outcomes, including time to flatus and to bowel movement are also favorable in our experience. Our study is limited with regards to the time period till discharge. As previously discussed, this has to do with the fact that patients are discharged after the removal of all the catheters and that the patients can remain in hospital until they are admitted to a rehabilitation center. The longer hospital stay can therefore not be used as a benchmark of the patients' general postoperative health or status.

We used the Clavien system of complication assessment and observed an overall complication rate of $49.4 \%$, with $11 \%$ of patients having a major complication (i.e. Clavien grade 3 or higher). Nevertheless, the rates and magnitude of these complications where on a par with those in well established open cystectomy literature (21-23).

This report has several important and noteworthy limitations. It fails to answer the question of whether robotic surgery is superior to conventional open surgery. In addition, this study does not evaluate long-term cancer related outcomes, e.g. 2-year and preferably 5-year results that remain the true benchmarks of oncological efficacy. Despite this, it is a relatively large case series examining the perioperative and pathological results of robotic surgery. 


\section{CONCLUSIONS}

Our experience with robotic-assisted laparoscopic radical cystectomy shows acceptable operative, pathological and short-term clinical outcomes. This suggests that robotic radical cystectomy has similar short-term cancer control and complication rates, less operative time and blood loss and earlier return to bowel function than laparoscopic or open radical cystectomy.

Certainly larger series are required to adequately evaluate and validate this procedure as an appropriate surgical and oncological option for patients with bladder cancer. Attention should however be drawn to the fact that these were the first cases ever performed at our center and certainly do include a form of learning curve. Considering this fact, the future of robotic assisted laparoscopic radical cystectomies seems extremely promising.

\section{CONFLICT OF INTEREST}

None declared.

\section{REFERENCES}

1. Guru KA, Kim HL, Piacente PM, Mohler JL: Robot-assisted radical cystectomy and pelvic lymph node dissection: initial experience at Roswell Park Cancer Institute. Urology. 2007; 69: 469-74.

2. Hemal AK: Robotic and laparoscopic radical cystectomy in the management of bladder cancer. Curr Urol Rep. 2009; 10: 45-54.

3. Pruthi RS, Wallen EM: Is robotic radical cystectomy an appropriate treatment for bladder cancer? Short-term oncologic and clinical follow-up in 50 consecutive patients. Urology. 2008; 72: 617-20; discussion 620-2.

4. Murphy DG, Challacombe BJ, Elhage 0, O'Brien TS, Rimington $\mathrm{P}$, Khan MS, et al.: Robotic-assisted laparoscopic radical cystectomy with extracorporeal urinary diversion: initial experience. Eur Urol. 2008; 54: 570-80.

5. Wang GJ, Barocas DA, Raman JD, Scherr DS: Robotic vs open radical cystectomy: prospective comparison of perioperative outcomes and pathological measures of early oncological efficacy. BJU Int. 2008; 101: 89-93.
6. Coward RM, Smith A, Raynor M, Nielsen M, Wallen EM, Pruthi RS: Feasibility and outcomes of robotic-assisted laparoscopic radical cystectomy for bladder cancer in older patients. Urology. 2011; 77: 1111-4.

7. Menon M, Shrivastava A, Kaul S, Badani KK, Fumo M, Bhandari $\mathrm{M}$, et al.: Vattikuti Institute prostatectomy: contemporary technique and analysis of results. Eur Urol. 2007; 51: 648-57; discussion 657-8.

8. Stein JP, Quek ML, Skinner DG: Lymphadenectomy for invasive bladder cancer. II. technical aspects and prognostic factors. BJU Int. 2006; 97: 232-7.

9. Dindo D, Clavien PA: What is a surgical complication? World J Surg. 2008; 32: 939-41.

10. Fueglistaler $P$, Adamina $M$, Guller U: Non-inferiority trials in surgical oncology. Ann Surg Oncol. 2007; 14: 1532-9.

11. Constantinides CA, Tyritzis SI, Skolarikos A, Liatsikos E, Zervas A, Deliveliotis C: Short- and long-term complications of open radical prostatectomy according to the Clavien classification system. BJU Int. 2009; 103: 336-40.

12. Skinner EC, Stein JP, Skinner DG: Surgical benchmarks for the treatment of invasive bladder cancer. Urol Oncol. 2007; 25: 66-71.

13. Herr H, Lee C, Chang S, Lerner S; Bladder Cancer Collaborative Group: Standardization of radical cystectomy and pelvic lymph node dissection for bladder cancer: a collaborative group report. J Urol. 2004; 171: 1823-8; discussion 1827-8.

14. Richards KA, Hemal AK, Kader AK, Pettus JA: Robot assisted laparoscopic pelvic lymphadenectomy at the time of radical cystectomy rivals that of open surgery: single institution report. Urology. 2010; 76: 1400-4.

15. Ng CK, Kauffman EC, Lee MM, Otto BJ, Portnoff A, Ehrlich $J R$, et al.: A comparison of postoperative complications in open versus robotic cystectomy. Eur Urol. 2010; 57: 274-81.

16. Haber GP, Gill IS: Laparoscopic radical cystectomy for cancer: oncological outcomes at up to 5 years. BJU Int. 2007; 100: 137-42.

17. Chang SS, Smith JA Jr, Wells N, Peterson M, Kovach B, Cookson MS: Estimated blood loss and transfusion requirements of radical cystectomy. J Urol. 2001; 166: 2151-4.

18. Manoharan M, Katkoori D, Kishore TA, Antebie E: Roboticassisted radical cystectomy and orthotopic ileal neobladder using a modified Pfannenstiel incision. Urology. 2011; 77: 491-3.

19. Tewari A, Jhaveri J, Rao S, Yadav R, Bartsch G, Te A, et al.: Total reconstruction of the vesico-urethral junction. BJU Int. 2008; 101: 871-7.

20. Khan MS, Elhage 0 , Challacombe B, Rimington P, Murphy D, Dasgupta P: Analysis of early complications of roboticassisted radical cystectomy using a standardized reporting system. Urology. 2011; 77: 357-62. 
21. Novotny V, Hakenberg OW, Wiessner D, Heberling U, Litz RJ, Oehlschlaeger S, et al.: Perioperative complications of radical cystectomy in a contemporary series. Eur Urol. 2007; 51: 397401; discussion 401-2.

22. Lowrance WT, Rumohr JA, Chang SS, Clark PE, Smith JA Jr, Cookson MS: Contemporary open radical cystectomy: analysis of perioperative outcomes. J Urol. 2008; 179: 1313-8; discussion 1318.
23. Frazier HA, Robertson JE, Paulson DF: Complications of radical cystectomy and urinary diversion: a retrospective review of 675 cases in 2 decades. J Urol. 1992; 148: 1401-5.

\section{Correspondence address} Dr. Adrian Treiyer University of Homburg Saarland, Kirrbergerstr. 8, Germany E-mail: aetreiyer@yahoo.com.ar 